\title{
APPLE MATURITY DISCRIMINATION AND POSITION
}

\author{
He Bei, Liu Gang* \\ Key Laboratory of Modern Precision Agriculture System Integration Research, China \\ Agricultural University, Beijing, China, 100083 \\ * Corresponding author, Address: P.O. Box 125, China Agricultural University, Qinghua \\ Donglu 17, Haidian District, Beijing, 100083, P. R. China, Tel: +86-10-62736741, Fax: +86- \\ 10-62736746,Email:pac@cau.edu.cn
}

Abstract: The most crucial problem of apple harvesting robot is to get the exact location and the maturity degree of the target. A system of apple maturity discrimination and positioning based on the multi-spectral technology and laser triangulation ranging principle is researched. Signal transmission, signal reception, signal processing, the CPU processing and several PC components are involved in the system. The distance between the apple and the device was calculated according to principle of triangulation. Ripe red apple, unripe green apple, stems and leaves have different reflectivity to red and infrared beam. The different ratio of red and infrared signal can be used to identify whether an apple is ripe. The threshold used to judge whether an apple is ripe is an experiential interval, which is acquired by several experiments.

Keywords: multi-spectral vision technology, triangle ranging, maturity of discrimination, positioning, nonlinear least square method

\section{INTRODUCTION}

Fruit harvesting is the most time-consuming and laborious part in fruit production chain. Because of its seasonal characteristic, labor intensity and high cost, ensuring harvesting fruit timely, lowering harvesting costs is an important way to increase agricultural income. Due to the complexity of harvesting operations, harvesting is still in a very low degree of automation. At present, domestic fruit harvesting operations is basically done by labor, 
and its harvest cost accounts for about 50\% - 70\% of the total cost. And the harvest time is concentrated. As an important agricultural robot types, harvesting robot is used to reduce the intensity of labor and production costs, improve labor productivity and product quality and ensure harvesting fruit timely, so it has great potential (Van Henten, 2003).

\section{SYSTEM DESIGN AND ANALYSIS}

\section{1 $\quad$ Functional module structure}

The system of apple maturity discrimination and positioning based on the multi-spectral technology and laser triangulation ranging principle included signal transmission, signal reception, signal processing, CPU and PC, as shown in the Fig. 1.

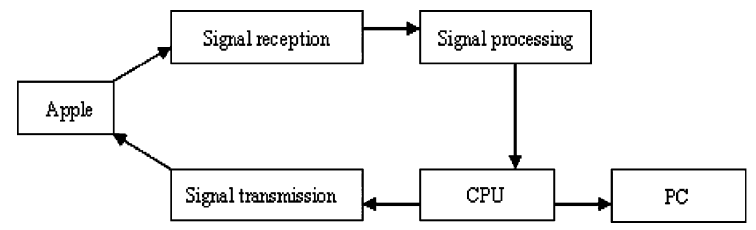

Fig. 1. General structure of the system

\subsection{System principle}

The system principle is shown in the Fig. 2.

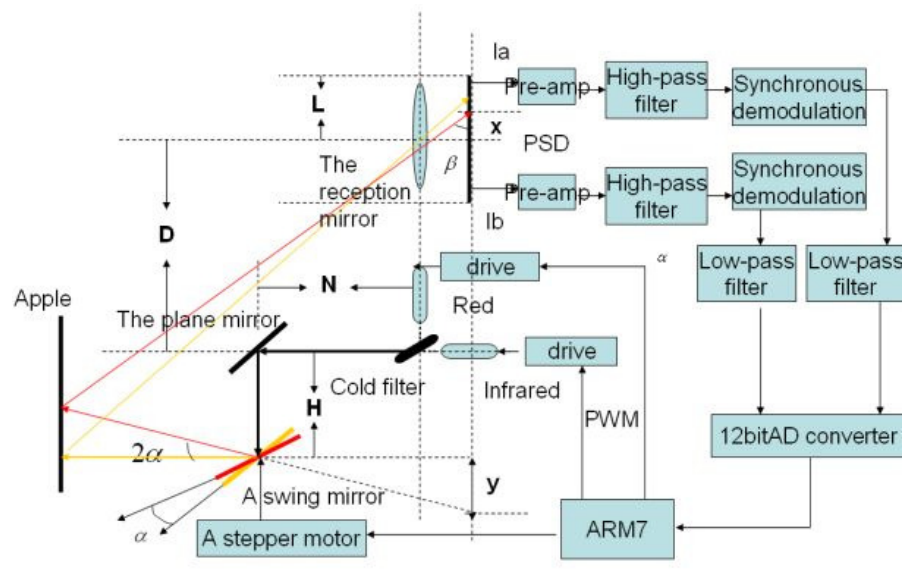

Fig. 2. System principle 
ARM7 outputted two PWM pulses, which respectively modulate two laser beams including a red laser beam $(685 \mathrm{~nm})$ and an infrared laser beam (830nm) (Liu Weiming, 2004). The two modulated beams go through a transmission lens and unite into one beam, then reflect by a fixed plane mirror. The united beam exposes to a rotatable swing lens, which is controlled by a stepper motor, and forms the linear scanning beam. Some beams reflected by apple go through the reception lens, and then focalize and image on the sensitive surface of semiconductor components PSD, which is a position sensitive sensors. PSD output two current associated with the photoelectric position. After the current-voltage conversion, amplification, filtering and demodulation etc., voltage signal was collected by ARM7 through 12 bit A/D converters. ARM7 processed the acquired data, based on the triangulation ranging principle and nonlinear least squares principle. The coordinates of the center of the apple was calculated, and then the maturity degree of the apple was judged, according to the acquired ratio of red signal and infrared signal. At last, ARM7 would transmit the processing results to the PC.

\section{SYSTEM HARDWARE DESIGN}

\subsection{Signal transmission and reception}

It was necessary to measure the reflectance characteristics of the apple for designing the system. This characteristic was measured by using a spectrum reflectance analyzer. The measurement result is shown in the Fig. 3.

This shows that the light beam with wavelength $685 \mathrm{~nm}$ was well reflected by the red apple fruit and was reflected not so much by the other part such as the leaf, green apple, and stem that contain much chlorophyll. The light beam with wavelength $830 \mathrm{~nm}$ was well reflected by all parts of the apple

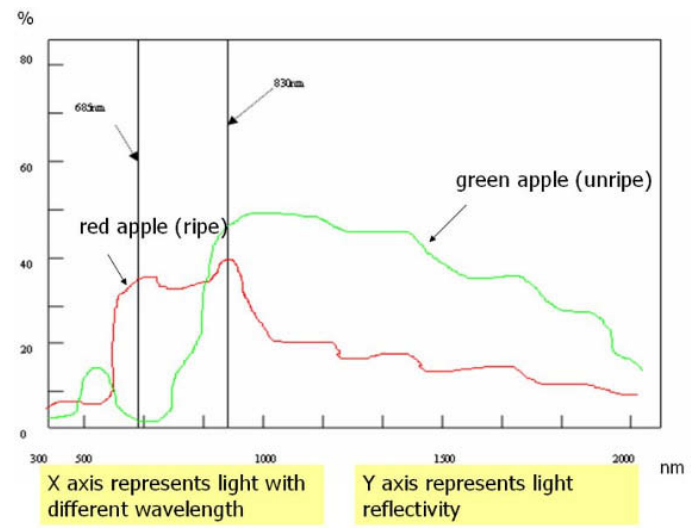

Fig. 3. Reflectance characteristic of apple 
plant. Based on this characteristic, the light transmission had two laser triode, one transmit red beam, which wavelength was $685 \mathrm{~nm}$; the other transmit infrared beams, which wavelength was $830 \mathrm{~nm}$. The intensity was $10 \mathrm{~mW}$.

PWM was used to control the laser transmitter to emit red and infrared beams. Two laser diodes were fixed perpendicularly. Two beams were concentrated into one optic axis by a cold filter. As shown in the Fig. 4.

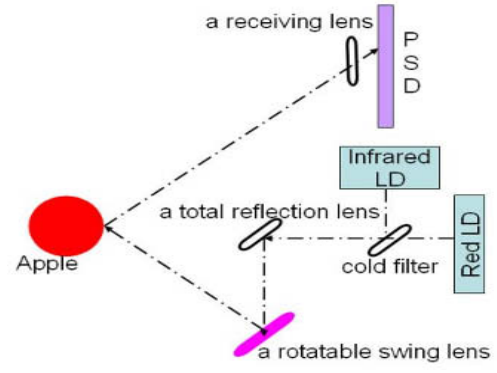

Fig. 4. Block diagram of signal transmission and reception

\subsection{Signal processing}

The object of this system was to detect the micron dimension motion. So the selection of device was very important (Pei Xiandeng, 2004).

AC modulation frequency method was used to separate background light from dark current. Choosing this method is for the reason that the disturbance of background light is always natural light and artificial light source, whose brightness changes slowly. The PSD response is direct current and low-frequency signal. If do high-frequency modulation to the object, the PSD response is high-frequency pulse signal. So high-pass filter can separate the signal and disturbance. To ensure the completion of position calculation and generate strong light intensity on PSD, square wave modulation and synchro position demodulation is adopted. This method can eliminate background light and dark-current effectively. The brightness of outside

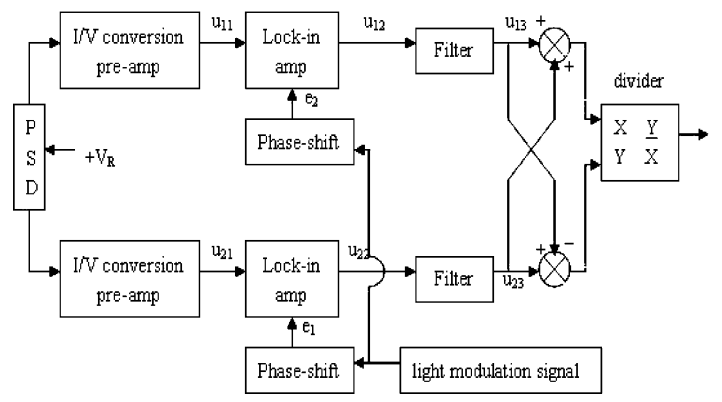

Fig. 5. Block diagram of modulation-demodulation 
light is almost unchangeable in this system. It is suitable to use modulation method to eliminate background light and dark-current. So this system takes the method as the signal processing part. As shown in the Fig. 5.

\section{SYSTEM SOFTWARE DESIGN}

\subsection{Main function}

The software system mainly had the following functions:

(1) Control the rotation and operation of the stepper motor

(2) Drive the laser diode fire red and infrared beams

(3) Collect AD signals

(4) Process data and transmit the distance and the maturity value to the PC

\subsection{Main program}

The main program is shown in the Fig. 6.

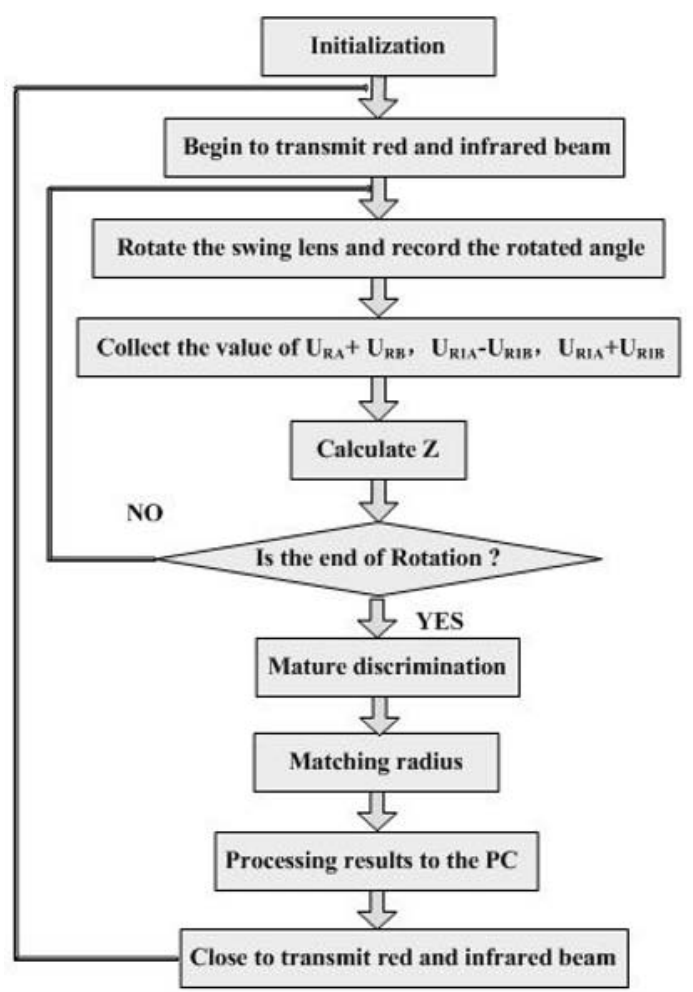

Fig. 6. Software flowchart 


\subsection{Maturity model}

The signal outputted by the PSD A, B pole went through synchronous detection, low pass filter and become red beam signal URA and URB and infrared signal URIA and URIB. As shown in the Fig. 7.

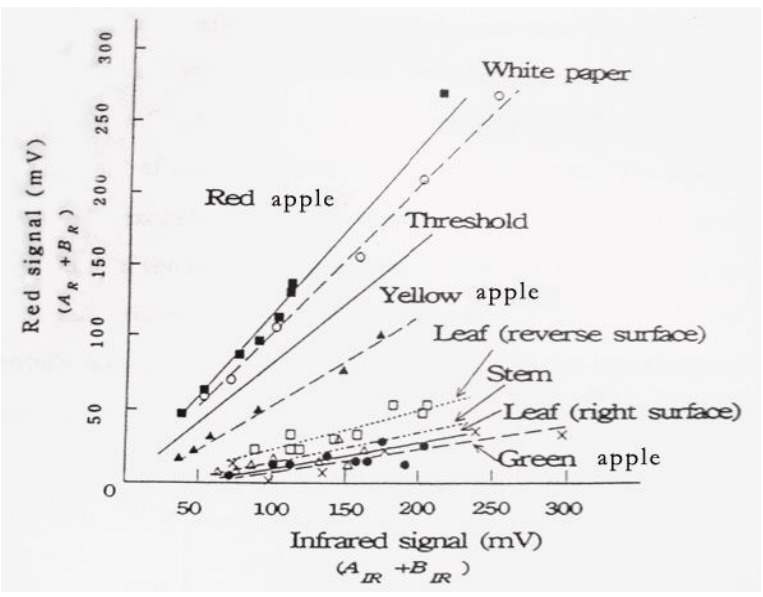

Fig. 7. Relationship between infrared and red signal

Through numerous experiments, and analysis of the literature found, using the ratio between the red signal and the infrared signals as the threshold value could extract mature fruits from others such as immature fruits, leaves, stems, and objects that were used to support the plant. Above figure shows that the ratios was small for green apples, leaf, and stem that contains a large amount of chlorophyll. For the apple, the ratio increased with the degree of maturity. Therefore, the red fruit could be extracted from the other parts of the plant by the ratio between the red and the infrared signals. However other objects such as white polypropylene rope and vinyl chloride pipe that were used to support the crop and gave high ratios that were nearly the same as that of the red fruit. Therefore, it was necessary to develop an algorithm for extracting such objects.

The formula to calculate the degree of maturity was URA+URB/ URIA+URIB.

\subsection{The calculation of the distance between the target apple and the device}

Supposing the length of PSD was 2L, and the output current of two poles of PSD were Ia and Ib. The distance between incident facular and the center of PSD was X. It could be concluded: 


$$
\mathrm{X}=\frac{\left(\mathrm{I}_{b}-\mathrm{I}_{a}\right) \times L}{\left(\mathrm{I}_{b}+\mathrm{I}_{a}\right)}
$$

As the figure 9 shows, the focus of transmission lens was $\mathrm{C}$, the distance between the transmission lens and total reflection lens was $\mathrm{N}$, the distance between the total reflection lens and the swing lens was $\mathrm{H}$, the distance between transmission lens and reception lens was D. When the swing lens rotated $\alpha$ degree, the beam scanned the region of apple was $2 \alpha$, the angle between the beam and the PSD was $\beta$. The distance y was as the following Fig. 8 shows.

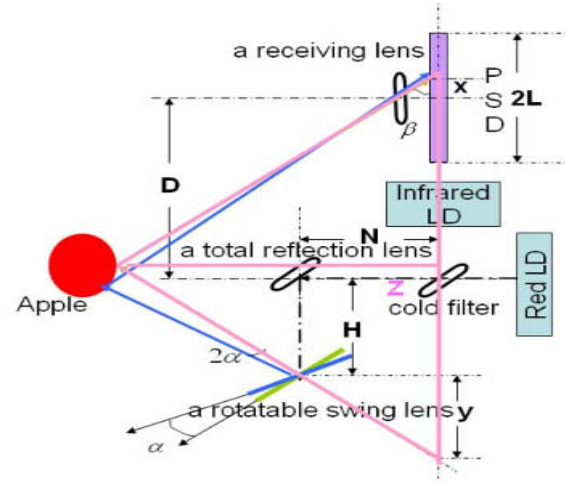

Fig. 8. Diagram of distance calculation

As the figure shows,

$$
\begin{aligned}
& \operatorname{tg} \beta=C / X \\
& \operatorname{tg} 2 \alpha=y / N \Rightarrow y=\operatorname{tg} 2 \alpha \times N \\
& \Rightarrow \frac{y+H+D+X}{\operatorname{Sin}(180-\beta-90+2 \alpha)}=\frac{\operatorname{tg} 2 \alpha \cdot N+H+D+X}{\operatorname{Sin}(90-\beta+2 \alpha)}=\frac{\operatorname{tg} 2 \alpha \cdot N+H+D+X}{\operatorname{Cos}(2 \alpha-\beta)}
\end{aligned}
$$

So the formula of calculation of distance between the apple and the device was:

$$
Z=\operatorname{Cos} 2 \alpha \cdot \operatorname{Sin} \beta \cdot \frac{\operatorname{tg} 2 \alpha \cdot N+H+D+\frac{\left(\mathrm{I}_{b}-\mathrm{I}_{a}\right) \times L}{\left(\mathrm{I}_{b}+\mathrm{I}_{a}\right)}}{\operatorname{Cos}(2 \alpha-\beta)}
$$

The actual distance between the apple and the sensor was:

$$
\mathrm{Z}_{S}=\operatorname{Cos} 2 \alpha \cdot \operatorname{Sin} \beta \cdot \frac{\operatorname{tg} 2 \alpha \cdot N+H+D+\frac{\left(\mathrm{I}_{b}-\mathrm{I}_{a}\right) \times L}{\left(\mathrm{I}_{b}+\mathrm{I}_{a}\right)}}{\operatorname{Cos}(2 \alpha-\beta)}+R
$$

where, $\mathrm{R}$ represents the radius of the apple, so the apple was almost round. According to round matching method, nonlinear least squares principle was used to calculate. Least squares principle was a mathematics optimization method. It found the best matching of an array by the least square error. 


\section{CONCLUSIONS}

The following conclusions are inferred from the study:

(1) A ranging method based on laser triangulation principle, which used to calculate the distance between the target apple and the device is designed. It uses specific wavelength light to line scanning the vision field, receive the reflected light by PSD photoelectric sensor, calculate the characteristic of receiving light, and get the distance between the target apple and the device at last.

(2) After analyzing many matching algorithm, linear least squares principle, which converted from the nonlinear least squares principle is used to implement matching calculation. This algorithm can give a best matching of the contour of the apple and get the radius of it.

(3) In the study of fruit maturity model, a new method is proposed. It is not identifying the maturity of fruit by texture characteristic, shape characteristic or turning the RGB color space to HIS, HSV, YIQ, color space. It is according to the different reflectivity of fruit and leaves of the crop to some specific wavelength light to identify the maturity of fruit. Compared with other method, spectrum method is more accurate and reliable.

\section{ACKNOWLEDGEMENTS}

This paper is supported by the national 863 projects: Key Technique of Fruit Harvesting Robot on Machine Vision (2006AA10Z255).

\section{REFERENCES}

Ahmad, I.S. \& J.F. Reid. 1996. Evaluation of Color Representations for Maize Images. Journal of Agricultural Engineering Research, 63(3):185-195.

D.M. Bulanon, T. Kataoka, S. Zhang. 2001. Optimal Thresholding for the Automatic Recognition of Apple Fruits. Transactions of the ASAE.

Liu Weiming, Mao Hanping, Wang Xinzhong. Application of Multi-spectrum Reflectance Vision Technique for Harvesting Robot [J]. Agricultural Equipment \& Technology, 2004, 30(6):15-18.

Pei Xiandeng, Luo Chun, HuangHa. Research of high precision position measurement system and its design based on PSD. Huazhong Univ. of Sci. \& Tech. (Nature Science Edition, 2004, 32(2):7-9.

Shigehiko Hayashi, Katsunobu Ganno, Yukitsugu Ishii, et al. Robotic harvesting system for eggplants [J]. JARQ, 2002, 36(3):163-168.

Van Henten E.J., Van Tuijl B.A.J., Hemming J. Field test of an autonomous cucumber picking robot [J]. Biosystems Engineering, 2003, 86(3):305-307.

Wang Xiaodong, Zhao Jie, Wu Wei. Measuring and Control of Laser Range Sensor Using PSD. Journal of Transducer Technology, 1995(5):37-38. 